\title{
Univalent functions in the Banach algebra of continuous functions
}

\author{
Yong Chan Kim and Jae Ho Choi ${ }^{2 *}$
}

\section{"Correspondence:}

choijh@dnue.ac.kr

${ }^{2}$ Department of Mathematics

Education, Daegu National

University of Education, Daegu,

705-715, Korea

Full list of author information is

available at the end of the article

\section{Abstract}

In this paper, we investigate several interesting properties of a composition operator defined on the open unit ball $B_{0}$ of the Banach algebra $C(T)$. We also consider the Noshiro-Warschawski theorem in the Banach algebra of continuous functions.

MSC: Primary 30C45; secondary 46J10

Keywords: analytic function; univalent function; Banach algebra;

Noshiro-Warschawski theorem

\section{Introduction and definitions}

Throughout this paper, $C(T)$ denotes the Banach algebra, with sup norm, of continuous complex-valued functions defined on a compact metric space $T$. Let $B(f: r)$ be an open ball in $C(T)$ centered at $f \in C(T)$ with radius $r$. In particular, for the sake of brevity, we use the simplified notation $B_{0}$ instead of $B(0: 1)$.

Let $\mathcal{A}$ denote the class of functions $\varphi(z)$ of the form

$$
\varphi(z)=z+\sum_{n=2}^{\infty} a_{n} z^{n}
$$

which are analytic in the open unit disk

$$
\mathcal{U}=\{z: z \in \mathbb{C} \text { and }|z|<1\} .
$$

Also, let $\mathcal{S}$ denote the class of all functions in $\mathcal{A}$ which are univalent in the unit disk $\mathcal{U}$. A function $\varphi(z)$ belonging to the class $\mathcal{S}$ is said to be convex in $\mathcal{U}$ if and only if

$$
\Re\left\{1+\frac{z \varphi^{\prime \prime}(z)}{\varphi^{\prime}(z)}\right\}>0 \quad(z \in \mathcal{U}) .
$$

We denote by $\mathcal{K}$ the class of all functions in $\mathcal{S}$ which are convex in $\mathcal{U}$.

Corresponding to the function $\varphi \in \mathcal{A}$, we define a composition operator $F_{\varphi}: B_{0} \rightarrow C(T)$ by

$$
F_{\varphi}(f)=\varphi \circ f=f+\sum_{n=2}^{\infty} a_{n} f^{n} .
$$


We denote by $S_{C}$ the class of all functions $F_{\varphi}$ which are injective in the open unit ball $B_{0}$. We note that Nikić ([1], Definition 2) defined a similar class $S_{C}$ without using the function $\varphi$. In this case, we cannot ensure the convergence of the series

$$
f+\sum_{n=2}^{\infty} a_{n} f^{n} .
$$

Now we let $G$ be an open nonempty subset of $C(T)$. A function $F: G \rightarrow C(T)$ is said to be $L$-differentiable at a point $f \in G$ if there exists $\lambda \in C(T)$ and a map $\eta$ defined in a ball $B(0: r)$ with values in $C(T)$ such that

$$
\lim _{h \rightarrow 0} \frac{\eta(h)}{\|h\|}=0
$$

and such that

$$
F(f+h)-F(f)=\lambda h+\eta(h)
$$

for all $h \in B(0: r)$. We call $\lambda$ the $L$-derivative of $F$ at $f$ and denote it by $F^{\prime}(f)$. From [1], we see that

$$
F_{\varphi}^{\prime}(f)=\varphi^{\prime} \circ f
$$

where $\varphi^{\prime}$ is a derivative of $\varphi$.

In the present paper, we investigate several geometric properties of the class $S_{C}$ associated with the theory of univalent functions.

\section{Geometric properties of the composition operator $F_{\varphi}$}

We begin by proving the following theorem.

Theorem $1 F_{\varphi} \in S_{C}$ if and only if $\varphi \in \mathcal{S}$.

Proof $(\Leftarrow)$ Suppose that $F_{\varphi}(f)=F_{\varphi}(g)$ for the functions $f$ and $g$ in $B_{0}$. Then it means that

$$
\varphi(f(t))=\varphi(g(t))
$$

for all $t \in T$. Since $\varphi$ is univalent, $f(t)=g(t)$ for all $t \in T$.

$(\Rightarrow)$ Let $\varphi\left(z_{1}\right)=\varphi\left(z_{2}\right)$ for $z_{1}$ and $z_{2}$ in $\mathcal{U}$. If we take the constant functions $f$ and $g$ such that $f=z_{1}$ and $g=z_{2}$, then it is obvious that

$$
f \in B_{0} \text { and } g \in B_{0} \text {. }
$$

Furthermore, from (1.2) it is easy to see that

$$
F_{\varphi}(f)=F_{\varphi}(g)
$$

Since $F_{\varphi}$ is injective, we have $f=g$. Hence we get $z_{1}=z_{2}$. This completes the proof of Theorem 1. 
By using Brange's theorem [2], we obtain the following.

\section{Corollary 1 If}

$$
F_{\varphi}(f)=f+\sum_{n=2}^{\infty} a_{n} f^{n} \in S_{C}
$$

then

$$
\left|a_{n}\right| \leq n
$$

Now we prove the Noshiro-Warschawski theorem ([3], Theorem 2.16) in the Banach algebra $C(T)$.

Theorem 2 If the L-derivative $F_{\varphi}^{\prime}(f)$ has a positive real part for all $f \in B_{0}$, then

$$
F_{\varphi} \in S_{C}
$$

Proof If $f_{1} \in B_{0}, f_{2} \in B_{0}$ and $f_{1} \neq f_{2}$, then there exists $t \in T$ such that

$$
f_{1}(t) \neq f_{2}(t) \text {. }
$$

By the hypothesis,

$$
\Re\left\{F_{\varphi}^{\prime}(f)\right\}>0
$$

for all $f \in B_{0}$. It follows from (1.3) that

$$
\Re\left\{\varphi^{\prime}(f(t))\right\}>0 \quad\left(f \in B_{0}: t \in T\right) .
$$

Since

$$
\varphi\left(f_{2}(t)\right)-\varphi\left(f_{1}(t)\right)=\int_{f_{1}(t)}^{f_{2}(t)} \varphi^{\prime}(x) d x=\left(f_{2}(t)-f_{1}(t)\right) \int_{0}^{1} \varphi^{\prime}\left(\lambda f_{2}(t)+(1-\lambda) f_{1}(t)\right) d \lambda
$$

and

$$
\lambda f_{2}(t)+(1-\lambda) f_{1}(t) \in B_{0},
$$

equations (2.1) and (2.3) imply that

$$
\varphi\left(f_{2}(t)\right) \neq \varphi\left(f_{1}(t)\right) .
$$

Hence

$$
F_{\varphi}\left(f_{1}(t)\right) \neq F_{\varphi}\left(f_{2}(t)\right)
$$

at $t \in T$, which shows that $F_{\varphi}$ is injective. 
Remark Since $T$ is compact, $\{f(t): t \in T\}$ is a closed proper subset of $\mathcal{U}$. Hence the condition (2.2) does not imply

$$
\Re\left\{\varphi^{\prime}(z)\right\}>0 \quad(z \in \mathcal{U}) .
$$

Next we obtain the following.

\section{Theorem 3 Let}

$$
\varphi(z)=\frac{z}{1-z} .
$$

Then

$$
\left\{F_{\varphi}(f): f \in B_{0}\right\}
$$

is a convex subset in $C(T)$.

Proof Assume that

$$
\alpha>0, \quad \beta>0 \quad \text { and } \quad \alpha+\beta=1 \text {. }
$$

For the functions $f$ and $g$ in $B_{0}$, we let

$$
u(t) \equiv \alpha F_{\varphi}(f(t))+\beta F_{\varphi}(g(t))
$$

and

$$
v(t) \equiv \frac{u(t)}{1+u(t)} .
$$

Then we have

$$
u(t)=\frac{v(t)}{1-v(t)}=F_{\varphi}(v(t)) .
$$

Since

$$
\begin{aligned}
1-|v(t)|^{2} & =1-v(t) \overline{v(t)} \\
& =1-\frac{u(t)}{1+u(t)} \frac{\overline{u(t)}}{1+\overline{u(t)}} \\
& =\frac{1}{1+\overline{u(t)}}(1+u(t)+\overline{u(t)}) \frac{1}{1+u(t)} \\
& =\frac{1+2 \Re\{u(t)\}}{1+|u(t)|^{2}}>0,
\end{aligned}
$$

the function $v$ belongs to $B_{0}$. Thus we have

$$
u=F_{\varphi}(v) \in\left\{F_{\varphi}(f): f \in B_{0}\right\} .
$$

This completes the proof of Theorem 3. 
We now recall that the function

$$
\varphi_{\eta}(z)=\frac{z}{1-\eta z} \quad(\eta \in \mathbb{C},|\eta|=1)
$$

is the well-known extremal function (see [3]) for the class $\mathcal{K}$ of convex functions. If we let

$$
\varphi(z)=\frac{z}{1-z}
$$

then we note that

$$
\varphi_{\eta}(z)=\eta^{-1} \varphi(\eta z)
$$

Making use of Theorem 3 and (2.4), we can derive the following.

Corollary 2 If $\varphi$ is an extreme point of $\mathcal{K}$, then

$$
\left\{F_{\varphi}(f): f \in B_{0}\right\}
$$

is a convex subset in $C(T)$.

It is well known that the sharp inequality

$$
\left|f^{(n)}(z)\right| \leq \frac{n !(n+|z|)}{(1-|z|)^{n+2}} \quad(n=1,2,3, \ldots)
$$

holds for every $f \in \mathcal{S}$ (see [3, p.70, Exercise 6]).

In view of the inequality (2.5), we have a generalization of [1, Theorem 2] as follows.

Theorem 4 If $f \in B_{0}$ and $\varphi \in \mathcal{S}$, then the nth $L$-derivative of $F_{\varphi}$ at $f$ satisfies

$$
\left\|F^{(n)}(f)\right\| \leq \frac{n !(n+\|f\|)}{(1-\|f\|)^{n+2}} .
$$

Remark The proof would run parallel to that of [1, Theorem 2] because there are many similarities. But, as we have seen in equation (1.2), we find it to be different from the definition of the class $S_{C}$, which was given by Nikić [1]. So, we include the proof of Theorem 4 .

Proof Applying (1.2) and (1.3), it is not difficult to show that

$$
F_{\varphi}^{(n)}(f)=\varphi^{(n)} \circ f \quad(n=1,2,3, \ldots),
$$

where $\varphi^{(n)}$ is the $n$th derivative of $\varphi$. Since

$$
F_{\varphi}^{(n)}(f) \in C(T)
$$

and $T$ is a compact metric space, there exists a point $\xi \in T$ such that

$$
\left\|F_{\varphi}^{(n)}(f)\right\|=\left|F_{\varphi}^{(n)}(f(\xi))\right|=\left|\varphi^{(n)}(f(\xi))\right| .
$$


Since $\varphi \in \mathcal{S}$, from (2.4) we have

$$
\left|\varphi^{(n)}(f(\xi))\right| \leq \frac{n !(n+|f(\xi)|)}{(1-|f(\xi)|)^{n+2}} \leq \frac{n !(n+\|f\|)}{(1-\|f\|)^{n+2}} .
$$

Combining (2.6) and (2.7), we obtain the desired result.

\section{Examples}

Example 1 Let the function $\varphi$ be defined by (1.1). For a fixed radius $0<r<1$, we let $T=$ $\{z \in \mathbb{C}:|z| \leq r\}$. If we define a continuous function $f: T \rightarrow \mathbb{C}$ by $f(z)=z$, then

$$
F_{\varphi}(f)=\varphi
$$

on $T$.

Example 2 Setting $\varphi(z)=z$ in (1.2), we have

$$
F_{\varphi}(f)=f
$$

Example 3 If $\varphi \in \mathcal{A}$ satisfies

$$
\Re\left\{\varphi^{\prime}(z)\right\}>0 \quad(z \in \mathcal{U}),
$$

then the Noshiro-Warschawski theorem implies that $\varphi$ is univalent. Hence, by Theorem 1 , we obtain

$$
F_{\varphi} \in S_{C}
$$

\section{Competing interests}

The authors declare that they have no competing interests.

\section{Authors' contributions}

All authors read and approved the final manuscript.

\section{Author details}

'Department of Mathematics Education, Yeungnam University, Gyongsan, 712-749, Korea. ²Department of Mathematics Education, Daegu National University of Education, Daegu, 705-715, Korea.

\section{Acknowledgements}

Dedicated to Professor Hari M Srivastava.

Received: 24 December 2012 Accepted: 7 March 2013 Published: 2 April 2013

\section{References}

1. Nikić, M: Koebe's and Bieberbach's inequalities in the Banach algebra of continuous functions. J. Math. Anal. Appl. 199, 149-156 (1996)

2. de Branges, L: A proof of the Bieberbach conjecture. Acta Math. 154, 137-152 (1985)

3. Duren, PL: Univalent Functions. A Series of Comprehensive Studies in Mathematics, vol. 259. Springer, Berlin (1983) 